migration for both these species. At least three Purple Finches hit picture windows and were brought for identification.

Bobolink-always scarce and locial, have been located in at least six areas within a $30-$ mile radius of Saskatoon. Largest colony- 12 males-6 miles north and 4 miles west of Saskatoon. B. Gollop.
Lark Bunting-first Saskatoon records. Five males near the Forestry Farm, June 8 and 10, 1960. B. Gollop. One on Highway \#5, 13 miles easit of city. R. Folker.

Chestnut-collared Longspur - first Saskatoon $r \in$ cord-small colony in pasture, just west of Burke Lake, 15 miles east of Saskatoon. Further study will be made of these birds. R. Folker, J. Hogg, F. Roy.

\title{
INFLUX OF LARK BUNTINGS AT HAWARDEN
}

\section{by Harold Kvinge, Hawarden}

We have had an influx of Lark Buntings into this area this summer. There are perhaps a dozen pairs just northwest of our buildings. I presume they are nesting as they have been around for some weeks, although I have not so far found any nests (up to July 15). This is the largest concenitration of these birds in this district since maybe 1937 when they were all around here.

EDITOR'S NOTE: The movement cf Lark Buntings northward into south-central Saskat-

\begin{abstract}
chewan during the past two years has been noted with interest by birders in various, districts. A year ago in the BIue Jay (XVII:129) we printed several reports of the increase of Lark Buntings in 1959 in areas where they had been scarce for some years. This year I have sean them ccmmonly around Regina where, until they reappeared in small number's !ast year, they have been more or less absent sin ze the thirties, at which time they were considered a common breeding resident. At Moose Jaw, too, I have ncted that they seem to be as common this year as during the thirties. North of Hawarden where Mr. Kvinge reports them as abundant, the Saskatoon Natural History Society is reporting Lark Buntings for the first time this year (see previous article in this issue by Frank Roy).
\end{abstract}

\section{A REPORT ON SPRING MIGRATION IN THE REGINA AREA, 1960}

Again this year members of the staff of the Saskatchewan Museum of Natural History and of the Bird Group of the Regina Natural History Society co-operated in a study of spring migration in the Regina area. Records submitted to the Museum and filed by Frank Brazier have been used as the basis for the following list of early arrival dates. A few additional dates were submitted to Margaret Belcher by members of the Bird Group and these have also been incorporated in the list.

\section{SPRING ARRIVAL DATES, 1960}

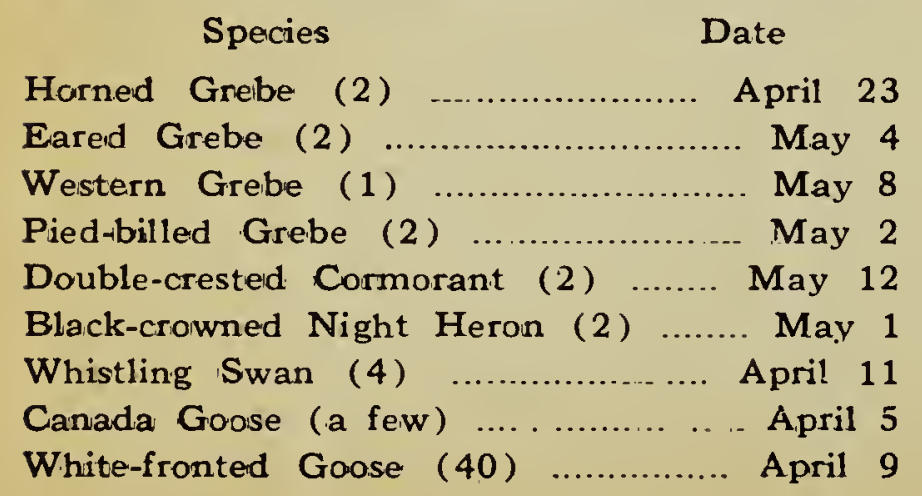

Snow Goose ............................. April 20 Blue Goose (2) ..................... April 9

Gadwall (4) ........................ April 23

Pintail (23) ............................ March 31

Green-winged Teal ........................ April 14

Blue-winged Teal (2) ................ April 21

American Widgeon ....................... April 7

Shcveler (6) ........................ April 10

Redhead. (2) ........................... April 10

Ring-necked Duck (2) ............... April 17

Canvasback (1) ...................... April 8

Lesser Scaup (14) .................... April 10

Common Goldeneye (5) ................. April 1

Bufflehead .............................. April 17

Camman Merganser (8) ............. March 31

Red-breasted Merganser (1) .......... April 10

Sharp-shinned Hawk (1) ............. April 12

Marsh Hawk (1) ....................... April 6

Cooper's Hawk (1) ........................ April 12

Red-tailed Hawk (1) .................... April 9

Brcad-winged Hawk (1) ................. May 3

Rough-legged Hawk (1) .............. March 27

Ferruginous Hawk (1) ..................... May 6

Bald Eagle (1) .......................... April 11

Sparrow Hawk (1) ....................... April 11

Sandhill Crane (50) .................... April 14

American Coot (1) ................ April 15 
Semipalmated Plover (1)

Killdeer

American Golden Plover

Black-bellied Plover (1)

Common Snipe (1)

Spotted Sandpiper (1)

Solitary Sandpiper (1)

Willet (1)

Greater Yellowlegs

(1)

Lesser Yellowlegs

Baind's Sandpiper (24)

Long-billed Dowitcher

Marbled Gcdwit (1)

Hudsonian Godwit (27)

American Avozet

Wilson's Phalarope (5)

Northern Phalarope (6)

Herring Gull (6)

Ring-billed Gull (7)

Franklin's Gull (2)

Bonaparte's Gull (5)

Forster's Tern

Common Tern (2)

Black Tern

(2)

Mourning Dove (2)

Burrowing Owl (4)

Short-eared Owl (1)

Common Nighthawk (8)

Belted Kingfisher (1)

Red-shafted Flicker (1)

Yellow-shafted Flicker (i)

Yellow-bellied Sapsucker (1)

Eastern Kingbird

Western Kingbird

Say's Phoebe

Least Flyclatcher (1)

Western Wood Pewee (1)

Horned Lark (3)

Tree Swallow (4)

4)

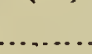

Roughwinged Swallow (1)

Barn Swallow (1)

Purple Martin (1)

Common Crow (12)

May 7

April 14

May 9

May 7

April 25

May 3

May 3

April 20

April 30

April 9

May 5

May 6

May. 6

April 28

April 23

April 23

May 10

May 14

April 10

April 15

April 21

May 4

May 6

Máy 7

May 9

April 16

May 6

April 16

May 28

April 22

April 10.

April 13

April 24

May 13

May 12

April 30

May 10

May 16

Jan. 31

April 25

May 19

May 5

May 6

March 30

Red-breasted Nuthatch (2) .............. May 3

Brown Creeper (1) ....................... April 26

House Wren (1) ............................ May 23

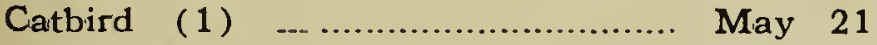

Brown Thrasher (1)

Robin (11) ............................. A pril 10

Hermit Thrush (1) ........................ April 24

Siwainson's Thrush (1) ..................... May 4

Gray-cheeked Thrush (4) ............... May 5

Mountain Bluebird (1) .................. March 26

Ruby-crowned Kinglet (1) ................. May 5

Water Pipit (2) .......................... May 1

Sprague's Pipit (a few) ............. April 16

Cedar Waxwing (2) .................... May 19

Lciggerhèad Shrike (1) .................. April 10

Solitary Vireo (2) .................... May 26

Red-eyed Vireo (1) ...................... May 16

Warbling Vireo (1) ...................... May 17

Tennessee Warbler (4) .................. May 17
Orange-crowned Warbler (1) _............. May 4

Yellow Warbler (1) ..................... May 11

Magnolia Warbler (3) .................. May 25

Myrtle Warbler (3) ................... April 25

Audubon's Warbler (1) ................. May 31

Blackpoll Warbler (3) ............... May 6

Palm Warbler ............................ May 15

Ovenbird (1) ........................ May 26

Northern Waterthrush (1) .......... May 26

Mcurning Warisler (2.) ................ May 2.5

Macgillivray's Warbler (1) ........... May 28

Yellowthroat (1) .......................... May 15

Wilson's Warbler (1) ................. May 24

American Pedistart (1) ................. May 17

Western Meadowlark (1) .............. April 1

Yellow-headed Blackbird ............. April 15

Redwinged Blackbird ..................... April 15

Baltimore Oriole ........................... May 16

Rusty Blackbird (4) ................ March 30

Brewer's Blackbird (several)....... April 26

Common Grackle ....................... April 18

Brown-headed Cowbird (1) .............. May 9

Rose-breasted Grosbeak (5) ........ May 17

Lazuli Bunting (1.2) ............ May 30

Purple Finch (3) .................... April 25

American Goldfinch (2) ................. May 21

Rufous-sided Torvhee (1) ............. May 8

Lark Bunting (1) ........................ May 21

Savannah Sparrow (1) ................. April 29

Vesper Sparrow (1) .................... April 20

Slate-colored Junco (2) .............. April 4

Oregon Junco (1) .......................... April 25

Tree Sparrow (1) ........................ March 26

Chipping Sparrow ('2) .................... May 10

Clay-colored Sparrow (2) .............. May 8

Harris' Sparrow ........................ May 10

White-crowned Sparraw (1) .............. May 2

White-throated Sparrow .................. April 26

Fiox ISparrow (4) ...................... April 25

Lincoln's Sparrow (8) ................. May 2

Song Sparrow (1) .................... April 10

Lapland Longspur ...................... April 4

Chestnut-colored Irongspur (2) ....... April 15

\section{NEST RECORD CARDS}

As soon as your summer nest record cards are completed please send them in so that a summary of nesting information for 1960 may be prepared for a repor' in the BLUE JAY. Cards should be sent to:

Prairie Nest Records Scheme, c/o Saskatchewan Museum of Natural History, Regina. 